\title{
DENOMINATOR SEQUENCES FOR CONTINUED FRACTIONS, III
}

\author{
R. T. WORLEY \\ To Professor K. Mahler on his seventy-fifth birthday
}

(Received 25 August 1977)

Communicated by J. H. Coates

\begin{abstract}
It is shown that for every irrational $\alpha$ the set of $\alpha^{\prime}$, for which $\alpha$ and $\alpha^{\prime}$ have infinitely many convergents with the same denominator, has the cardinality of the continuum.
\end{abstract}

Subject classification (Amer. Math. Soc. (MOS), 1970): 10 A 30.

For given real irrational $\alpha$, let the sequence of the denominators of the convergents of the simple continued fraction for $\alpha$ be called the denominator sequence of $\alpha$. By a result of Schmidt (1967) it follows that if $\alpha$ and $\alpha^{\prime}$ are two algebraic irrationals whose denominator sequences have a common subsequence then $\alpha, \alpha^{\prime}$ and 1 are linearly dependent over the rationals. In a recent paper (Worley, 1973) it was shown that the same conclusion held for $\alpha$ and $\alpha^{\prime}$ not restricted to be algebraic provided that the common subsequence $\left(B_{n}\right)$ is the denominator sequence for a third number $\alpha^{\prime \prime}$ and $B_{n}^{2}>\frac{1}{2} B_{n+1}$ for infinitely many $n$. The main result of this paper is in the opposite direction, namely:

THEOREM. For every real irrational $\alpha$ the set of $\alpha^{\prime}$ for which $\alpha$ and $\alpha^{\prime}$ have a common denominator subsequence has the cardinality of the continuum.

From this result it trivially follows that there is an $\alpha^{\prime}$ such that $\alpha^{\prime}, \alpha$ and 1 are not linearly dependent over the rationals. The proof of the theorem is based on the following lemma which in itself is of interest. 
Lemma 1. Let $a$ and $b$ be natural numbers with $(a, b)=1$. Then there exists an integer $n_{0}=n_{0}(a, b)$ such that every integer $n>n_{0}$ can be written as $n=a x+b y$ with $x, y$ integers such that $x>y>0$ and $(x, y)=1$.

Proof. Considering the congruence classes $\bmod a$ of $y b$ for $0 \leqslant y<a$ it is clear that $n=a x_{0}+b y_{0}$ where $0 \leqslant y_{0}<a$. We let $r$ denote the greatest integer less than $\left(x_{0}-y_{0}\right) /(a+b)$, and take the representations $n=a x_{l}+b y_{l}$ for $l=1,2, \ldots, r$, where $x_{l}=x_{0}-l b, y_{l}=y_{0}+l a$. The lemma is equivalent to the statement that if $n$ is sufficiently large the sum

$$
N=\sum_{\substack{1 \leq 1 \leq r \\ \delta_{l}=1}} 1,
$$

where $\delta_{l}=\left(x_{l}, y_{l}\right)$, is non-zero. We show by a simple sieve argument that $N>0$ for $n>c[a(a+b)]^{1+\varepsilon}$, where $c$ is a constant depending on the choice of $\varepsilon>0$.

We have

$$
\begin{aligned}
N & =\sum_{1 \leqslant \leqslant \leqslant r} \sum_{d \mid \delta_{l}} \mu(d) \\
& =\sum_{d \mid n} \mu(d) \sum_{1 \leq \leqslant r} \\
& =\sum_{d \mid n} \mu(d)\left(r d^{-1}+O(1)\right)
\end{aligned}
$$

since the $l$ for which $d \mid \delta_{l}$ form a unique congruence class mod $d$. The main term is clearly $r \varphi(n) / n$ and the error term is certainly less than $d(n)=\sum_{d \mid n} 1$.

Take an arbitrary $\varepsilon>0$ and let $\rho$ be such that $1 /(1-2 \rho)=1+\varepsilon$. By well-known estimates for $\varphi(n)$ and $d(n)$ we know there exist constants $c^{\prime}$ and $c^{\prime \prime}$ such that $\varphi(n)>c^{\prime} n^{1-\rho}$ and $d(n)<c^{\prime \prime} n^{\rho}$. Furthermore, if we assume $n>4 a(a+b)$ we have $r>n / 2 a(a+b)$. Hence

$$
N>c^{\prime} n^{1-\rho} / 2 a(a+b)-c^{\prime \prime} n^{\rho}
$$

and so $N>0$ provided $n^{1-2 \rho}>2 c^{\prime \prime} a(a+b) / c^{\prime}$, that is, $n>c[a(a+b)]^{1+\varepsilon}$ for a suitable constant $c$. It should be noted that this is compatible with our assumption $n>4 a(a+b)$ providing $c>4$.

Lemma 2. Let $S$ denote an infinite subset of the natural numbers, containing at least two relatively prime integers. Then there exist uncountably many distinct real numbers $\beta$ for which the denominator sequence contains infinitely many elements of $S$.

Proof. We first note that if $(a, b)=1$ and $a>b>0$ then there exists a rational number $r(a, b)=\left\langle 0, c_{1}, \ldots, c_{m}\right\rangle$ which has $b$ and $a$ as its final two denominators, 
where $\left\langle c_{0}, \ldots, c_{m}\right\rangle$ denotes the simple continued fraction

$$
c_{0}+\frac{1}{c_{1}}+\frac{1}{c_{2}}+\ldots+\frac{1}{c_{m}}
$$

Secondly we note that if $n$ can be expressed as $x a+y b$ where $(x, y)=1$ and $x>y>0$ and if $r(x, y)=\left\langle 0, d_{1}, \ldots, d_{p}\right\rangle$ then $n$ is the final convergent of

$$
\left\langle 0, c_{1}, \ldots, c_{m}, d_{1}, \ldots, d_{p}\right\rangle
$$

The proof is basically just the following procedure. Take two relatively prime elements $a, b \in S$. Using Lemma 1 there exists $n_{3} \in S$ with $n_{3}=a x+y b$ where $(x, y)=1$ and $x>y>0$. Hence there is a rational number $r_{3}=r\left(a, b, n_{3}\right)$ with $a, b$ ănd $n_{3}$ included among its denominators. Now let $b_{2}, a_{2}$ denote the final two denominators of $r\left(a, b, n_{3}\right)$, and repeat the above procedure to get $r_{4}=r\left(a, b, n_{3}, n_{4}\right)$ a rational number for which the continued fraction is an extension of the continued fraction for $r\left(a, b, n_{3}\right)$ and such that $r\left(a, b, n_{3}, n_{4}\right)$ has four elements $a, b, n_{3}$ and $n_{4}$ of $S$ among its denominators. If this procedure is repeated indefinitely the sequence $r_{3}, r_{4}, \ldots$ clearly converges to a number $\beta$ which has the desired property. A slight modification of this procedure ensures a choice at each step, so leading to uncountably many $\beta$ with the desired property. The exact details are as follows.

Let $a, b \in S$ be such that $(a, b)=1$ and $a>b$. We choose from $S$ a sequence $s_{0}, s_{1}, \ldots$ with the property $s_{0}=a, s_{m+1}>\max \left\{n_{0}(c, d): c<d \leqslant s_{m}+1\right\}$ for $m \geqslant 1$.

Choose an arbitrary sequence $\left(\varepsilon_{i}\right)_{i \in N}$ with the following properties.

(i) $\varepsilon_{1}=\varepsilon_{2}=1$,

(ii) $\varepsilon_{i}=0$ for infinitely many $i$, and

(iii) for each $i, \varepsilon_{i}=0$ or 1 .

The set of such sequences clearly has the cardinality of the continuum. For each such sequence we construct a number $\beta$ such that $s_{i}$ is a denominator of $\beta$ if $\varepsilon_{i}=0$ and $s_{i}+1$ is a denominator of $\beta$ if $\varepsilon_{i}=1$. This ensures that for $i \geqslant 3, s_{i}$ is a denominator of $\beta$ if and only if $\varepsilon_{i}=0$ and so the $\beta$ corresponding to distinct sequences are distinct. To see that $s_{i}$ is not a denominator of $\beta$ if $\varepsilon_{i}=1$ and $i \geqslant 3$ it is necessary to observe that consecutive integers are denominators only for $\beta=\langle 0,2, \ldots\rangle$ which has consecutive denominators 1,2 and for $\beta=\langle 0,1, q, \ldots\rangle$ which has consecutive denominators $q, q+1$.

We define $\beta$ to be the limit of the sequence $r_{0}, r_{1}, r_{2}, \ldots$ of rationals constructed inductively as follows. Firstly, we set $r_{0}=b / a$. Secondly, if $r_{j}$ has been constructed we let $r_{j}=\left\langle 0, c_{1}, \ldots, c_{q}\right\rangle$ have last two convergents $e$ and $f$, where $f \leqslant s_{j}+1$. If $\varepsilon_{j+1}=0$ we set $v_{j+1}=s_{j+1}$, while if $\varepsilon_{j+1}=1$ we set $v_{j+1}=s_{j+1}+1$. By the choice of $s_{j+1}$ we can write

$$
v_{j+1}=f y+e x
$$


where $x>y>0$ and $(x, y)=1$. We now set

$$
r_{j+1}=\left\langle 0, c_{1}, \ldots, c_{q}, d_{1}, \ldots, d_{k}\right\rangle
$$

where $y / x=\left\langle 0, d_{1}, \ldots, d_{k}\right\rangle$, so that the final denominator of $r_{j+1}$ is $v_{j+1}$. Plainly the sequence $\left(r_{j}\right)$ converges to a number $\beta$ with the desired property. As noted above, the numbers $\beta$ corresponding to different sequences $\left(\varepsilon_{i}\right)$ are distinct and so there are uncountably many such $\beta$.

ProOF OF THE THEOREM. The theorem follows from Lemma 2 on taking $S$ to be the denominator sequence of the given number $\alpha$.

It will be noted that if $\left(B_{n}\right)$ denotes the common denominator sequence to $\alpha$, and $\alpha^{\prime}$ is constructed as in Lemma 2 , then we have $B_{n+1}>c\left[B_{n}\left(B_{n}+b\right)\right]^{1+e}$ where $b<B_{n}$. Hence, regardless of the values chosen for $\varepsilon$ and $c$, we must have $B_{n+1} \geqslant 2 B_{n}^{2}$ for all but finitely many $n$. In other words, the condition $B_{n}^{2}>\frac{1}{2} B_{n+1}$ for finitely many $n$ cannot hold.

It will also be noted that the exact choice of $r_{0}$ in the proof of Lemma 2 is not critical to the conclusion of the theorem. Hence we can modify the proofs to show the existence of uncountably many $\alpha^{\prime}$, satisfying the conditions of the theorem, within any internal of the form $(a / b,(a+1) / b)$.

\section{References}

W. M. Schmidt (1967), "On simultaneous approximations of two algebraic numbers by rationals", Acta Math. 119, 27-50.

R. T. Worley (1973), “Denominator sequences of continued fractions.', J. Austral. Math. Soc. 15, 112-116.

Department of Mathematics

Monash University

Clayton, Victoria 3168

Australia 